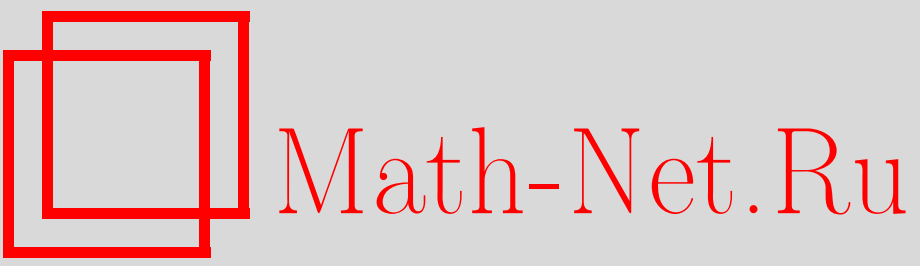

Т. А. Жильников, М. С. Маскина, Приложение преобразования Радона векторных функций к магнитному неразрушающему контролю, Итоги науки и техн. Сер. Соврем. мат. и ее прил. Темат. обз., 2020, том 182, 28-32

DOI: https://doi.org/10.36535/0233-6723-2020-182-28-32

Использование Общероссийского математического портала Math-Net.Ru подразумевает, что вы прочитали и согласны с пользовательским соглашением

http://www.mathnet.ru/rus/agreement

Параметры загрузки:

IP: 54.224 .135 .184

26 апреля 2023 г., 18:16:59 


\title{
ПРИЛОЖЕНИЕ ПРЕОБРАЗОВАНИЯ РАДОНА ВЕКТОРНЫХ ФУНКЦИЙ К МАГНИТНОМУ НЕРАЗРУШАЮЩЕМУ КОНТРОЛЮ
}

\author{
(c) 2020 г. $\quad$ Т. А. ЖКИЛЬНИКОВ, М. С. МАСКИНА
}

\begin{abstract}
АннотАция. В работе рассматривается преобразование Радона для векторных представлений функций. Предложена реализация неразрушающего магнитоиндукционного исследования, позволяющая получить изображение внутренней структуры намагниченного образца посредством измерения плотности пронизывающего его потока.
\end{abstract}

Ключевые слова: компьютерная томография, прямое преобразование Радона, обратное преобразование Радона, магнитный неразрушающий контроль, плотность магнитного потока.

\section{APPLICATION OF THE RADON TRANSFORM OF VECTOR FUNCTIONS TO MAGNETIC NONDESTRUCTIVE TESTING}

\author{
(c) 2020 T. A. ZHILNIKOV, M. S. MASKINA
}

\begin{abstract}
In this paper, we consider the Radon transform for vector representations of functions. We propose an implementation of a nondestructive magnetic-induction research, which allows one to obtain an image of the internal structure of a magnetized sample by measuring the density of the flux penetrating it.
\end{abstract}

Keywords and phrases: computed tomography, direct Radon transform, inverse Radon transform, magnetic nondestructive testing, magnetic flux density.

AMS Subject Classification: 53A45

1. Введение. Анализ известных методов магнитного неразрушающего контроля (НK) объектов закономерно указывает на необходимость использования метода компьютерной томографии, который базируется на частном случае преобразования Радона и представляет собой математический аппарат получения изображения распределения физической величины в объеме (см. [6]). Как следует из значения слова «томографический» в названии, данный метод представляет исследуемое трехмерное пространство объема с объектом внутри совокупностью сечений, благодаря чему размерность задачи понижается до двумерной (томографической), что допускает использование самого простого частного описания математического аппарата преобразования Радона, в котором интегрирование осуществляется по лучу и, как следствие, решается основное интегральное уравнение первого порядка (см. [1]). Возникающие при таком томографическом описании проблемы реализации магнитного неразрушающего контроля заключаются, во-первых, в том, что на практике измерение величины магнитной индукции (или, другими словами, плотности потока) возможно только через процедуру регистрации магнитного потока, которая предполагает интегрирование по площади, а не по прямой, а во-вторых, метод компьютерной томографии предполагает визуализацию скалярных распределений, в то время как магнитная индукция является 
распределением векторной величины. Таким образом, изложенным обусловлена потребность в отходе от частного описания математического аппарата преобразования Радона и использование его общего вида.

2. Прямое векторное преобразование Радона от функции трех переменных. Пусть $\boldsymbol{f}$ - исходная векторная функция со скалярными компонентами, во-первых, удовлетворяющими условиям регулярности, определенными в ограниченной области, а во-вторых, достаточно быстро убывающими к границам области. Координаты точек в трехмерном пространстве ограниченной области определения исходной векторной функции заданы в декартовой (прямоугольной) системе координат радиус-вектором $\boldsymbol{r}=(x, y, z)$, из начала координат, поэтому компоненты $\boldsymbol{f}$ в разложении вектора по ортогональному базису:

$$
\boldsymbol{f}(\boldsymbol{r})=f_{x}(\boldsymbol{r}) \cdot \boldsymbol{i}+f_{y}(\boldsymbol{r}) \cdot \boldsymbol{j}+f_{z}(\boldsymbol{r}) \cdot \boldsymbol{k},
$$

где $f_{x}(\boldsymbol{r}), f_{y}(\boldsymbol{r}), f_{z}(\boldsymbol{r})$ - скалярные компоненты векторной функции $\boldsymbol{f}(\boldsymbol{r})$, а $\boldsymbol{i}, \boldsymbol{j}, \boldsymbol{k}$ - ортогональный базис. Геометрический смысл векторного преобразования Радона $\boldsymbol{p}$ в трехмерном случае есть интеграл от исходной векторной функции $\boldsymbol{f}$ по гиперплоскости, перпендикулярной вектору нормали $\boldsymbol{n}$ и проходящей на расстоянии $s$ от начала координат (так, для трехмерного пространства гиперплоскость есть плоскость $\Omega$; см. [2]).

Интеграл по гиперплоскости, реализуемый в прямом векторном преобразовании Радона $\boldsymbol{p}$, определен с помощью дельта-функции Дирака через интеграл по всему пространству $\mathbb{R}^{3}$ :

$$
\boldsymbol{p}(s, \boldsymbol{n})=\int_{\mathbb{R}^{3}} \boldsymbol{f}(\boldsymbol{r}) \cdot \delta(s-(\boldsymbol{r} \cdot \boldsymbol{n})) d \boldsymbol{r}=p_{x}(s, \boldsymbol{n}) \cdot \boldsymbol{i}+p_{y}(s, \boldsymbol{n}) \cdot \boldsymbol{j}+p_{z}(s, \boldsymbol{n}) \cdot \boldsymbol{k},
$$

где $\delta$-дельта-функции Дирака, $s$-расстояние от начала координат до гиперплоскости, равное скалярному произведению векторов $s=(\boldsymbol{r} \cdot \boldsymbol{n}), d \boldsymbol{r}$ - трехмерный элемент объема, равный $d \boldsymbol{r}=d x d y d z$. Фактически каждая компонента векторной функции $\boldsymbol{p}(s, \boldsymbol{n})$ является линейной проекцией «плоскостной суммы значений» соответствующей компоненты исходной функции $\boldsymbol{f}$, определенная по оси $O S$ аргументом $s$ для каждого положения нормали $\boldsymbol{n}$ (см. рис. 1$)$. Кроме того, вектор нормали $\boldsymbol{n}$ коллинеарен единичному вектору оси $O S$.

Если исходная векторная функция действительных переменных определена на всей плоскости и достаточно быстро убывает на бесконечности (так что соответствующие несобственные интегралы сходятся), то в трехмерном случае преобразование Радона обратимо. В подтверждение этого рассмотрим векторную спектральную функцию $\boldsymbol{P}(\omega, \boldsymbol{n})$ пространственной частоты $\omega$ как одномерное преобразование Фурье $\boldsymbol{p}(s, \boldsymbol{n})$ по переменной $s$ :

$$
\boldsymbol{P}(\omega, \boldsymbol{n})=\int \boldsymbol{p}(s, \boldsymbol{n}) e^{-i \omega s} d s=P_{x}(\omega, \boldsymbol{n}) \cdot \boldsymbol{i}+P_{y}(\omega, \boldsymbol{n}) \cdot \boldsymbol{j}+P_{z}(\omega, \boldsymbol{n}) \cdot \boldsymbol{k} .
$$

Итак,

$$
\boldsymbol{P}(\omega, \boldsymbol{n})=\int\left[\int \boldsymbol{f}(\boldsymbol{r}) \cdot \delta(s-(\boldsymbol{r} \cdot \boldsymbol{n})) d \boldsymbol{r}\right] e^{-i \omega s} d s .
$$

Независимость векторной функции $\boldsymbol{f}$ от переменной интегрирования $s$ позволяет изменить очередность интегрирования:

$$
\boldsymbol{P}(\omega, \boldsymbol{n})=\int \boldsymbol{f}(\boldsymbol{r})\left[\int \delta((\boldsymbol{r} \cdot \boldsymbol{n})-s) e^{-i \omega s} d s\right] d \boldsymbol{r} .
$$

Из последнего выражения после свертки с дельта-функцией получаем

$$
\int \delta((\boldsymbol{r} \cdot \boldsymbol{n})-s) e^{-i \omega s} d s=e^{-i \omega(\boldsymbol{r} \cdot \boldsymbol{n})}
$$

тогда

$$
\boldsymbol{P}(\omega, \boldsymbol{n})=\int \boldsymbol{f}(\boldsymbol{r}) e^{-i \omega(\boldsymbol{r} \cdot \boldsymbol{n})} d \boldsymbol{r}
$$




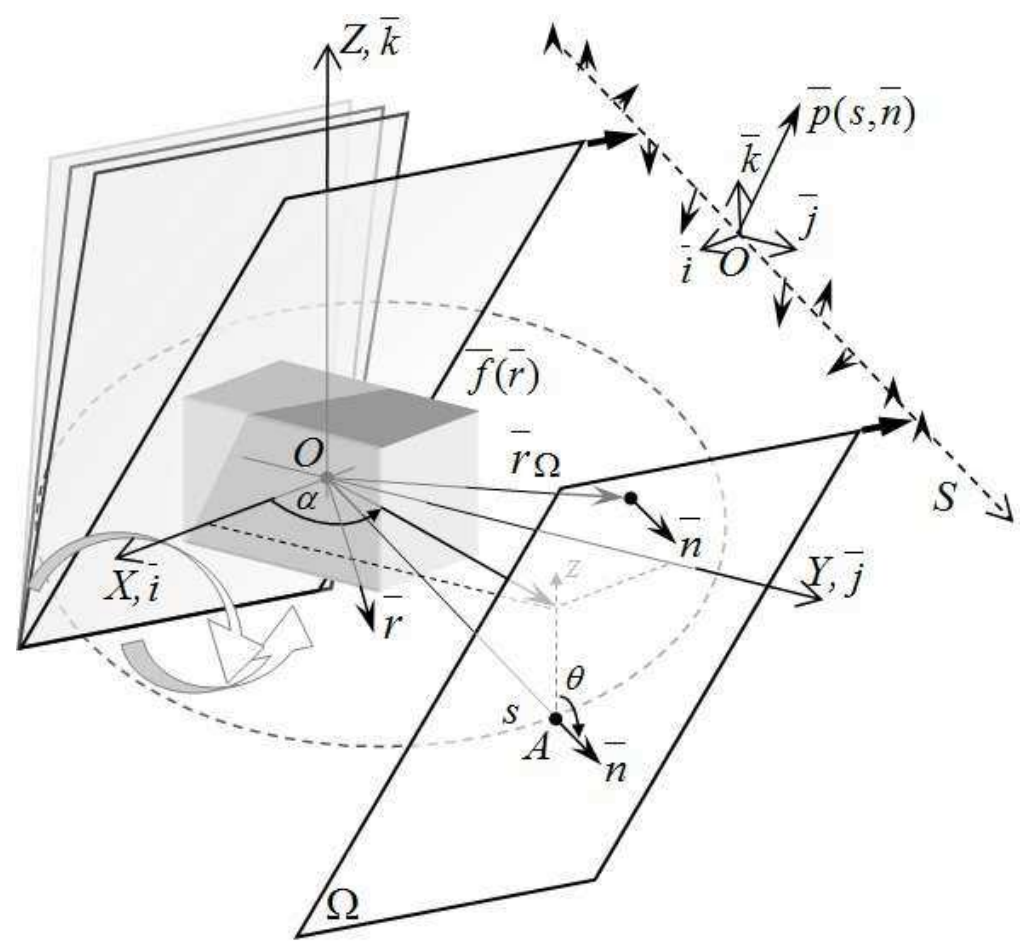

Рис. 1. Схема прямого векторного преобразования Радона

3. Обратное векторное преобразование Радона от функции трех переменных. Возможность возврата к исходной векторной функции посредством обратного преобразования Радона требует введения нового обозначения радиуса-вектора $\boldsymbol{r}^{\prime}$, определенного сверткой с дельтафункцией Дирака:

$$
\boldsymbol{f}\left(\boldsymbol{r}^{\prime}\right)=\int \boldsymbol{f}(\boldsymbol{r}) \cdot \delta\left(\boldsymbol{r}^{\prime}-\boldsymbol{r}\right) d \boldsymbol{r}
$$

Допустимо представить дельта-функцию в последнем выражении обратным Фурье-преобразованием ее многомерного изображения:

$$
\delta\left(\boldsymbol{r}^{\prime}-\boldsymbol{r}\right)=\frac{1}{(2 \pi)^{3}} \int e^{i \boldsymbol{\omega}\left(\boldsymbol{r}^{\prime}-\boldsymbol{r}\right)} d \boldsymbol{\omega}, \quad \boldsymbol{\omega}=\left(\omega_{x}, \omega_{y}, \omega_{z}\right)=\omega \cdot \boldsymbol{n},
$$

где $\omega=\sqrt{\omega_{x}^{2}+\omega_{y}^{2}+\omega_{z}^{2}}, \boldsymbol{n}=(\cos \alpha \cdot \sin \theta, \sin \alpha \cdot \sin \theta, \cos \theta), \theta$ и $\alpha$ - соответственно зенитный и азимутальный углы сферической системы координат, $d \boldsymbol{\omega}=d \boldsymbol{\omega} d \boldsymbol{n}$. Перейдем к сферической системе координат с радиус-вектором в области изображения, параметризованным как $\boldsymbol{\omega}=(\omega, \theta, \alpha)$, учитывая, что якобиан равен $|J|=\omega^{2} \cdot \sin \theta$ и $d \boldsymbol{n}=d \theta d \alpha$ :

$$
\delta\left(\boldsymbol{r}^{\prime}-\boldsymbol{r}\right)=\frac{1}{(2 \pi)^{3}} \iiint e^{i \omega\left(\boldsymbol{r}^{\prime}-\boldsymbol{r}\right) \boldsymbol{n}} \omega^{2} \cdot \sin \theta d \omega d \alpha d \theta
$$

Получим

$$
\boldsymbol{f}\left(\boldsymbol{r}^{\prime}\right)=\int \boldsymbol{f}(\boldsymbol{r})\left[\iint \frac{\omega^{2}}{(2 \pi)^{3}} \cdot \sin \theta e^{i \omega\left(\boldsymbol{r}^{\prime}-\boldsymbol{r}\right) \boldsymbol{n}} d \omega d \boldsymbol{n}\right] d \boldsymbol{r} .
$$

В последнем выражении раскрыты скобки в показателе экспоненциальной функции и изменена последовательность интегрирования:

$$
\boldsymbol{f}\left(\boldsymbol{r}^{\prime}\right)=\iint \frac{\omega^{2}}{(2 \pi)^{3}} e^{i \omega\left(\boldsymbol{r}^{\prime} \cdot \boldsymbol{n}\right)}\left[\int \boldsymbol{f}(\boldsymbol{r}) e^{-i \omega(\boldsymbol{r} \cdot \boldsymbol{n})} d \boldsymbol{r}\right] \sin \theta d \omega d \boldsymbol{n}
$$

где $\left(\boldsymbol{r}^{\prime} \cdot \boldsymbol{n}\right)$ и $(\boldsymbol{r} \cdot \boldsymbol{n})$ - скалярные произведения векторов. Учитывая, что интеграл в квадратных скобках есть спектральная векторная функция $\boldsymbol{P}(\boldsymbol{\omega}, \boldsymbol{n})$, которая определена соотношением $s=$ 
$(\boldsymbol{r} \cdot \boldsymbol{n})$, свяжем функциональным соотношением векторное преобразование Радона $\boldsymbol{p}$ в трехмерном случае и исходную векторную функцию $\boldsymbol{f}$ :

$$
\begin{aligned}
\boldsymbol{f}\left(\boldsymbol{r}^{\prime}\right)=\int\left(\int\left[\frac{\omega^{2}}{(2 \pi)^{3}}\right] \cdot\left[\int \boldsymbol{p}(s, \boldsymbol{n}) e^{-i \omega s} d s\right] e^{i \omega\left(\boldsymbol{r}^{\prime} \cdot \boldsymbol{n}\right)} d \omega\right) \sin \theta d \boldsymbol{n}= \\
=\int\left(\int H(\omega) \cdot \boldsymbol{P}(\omega, \boldsymbol{n}) e^{i \omega\left(\boldsymbol{r}^{\prime} \cdot \boldsymbol{n}\right)} d \omega\right) \sin \theta d \boldsymbol{n}
\end{aligned}
$$

где скалярная спектральная функция $H(\omega)=\omega^{2} /(2 \pi)^{3}-$ Фурье-образ свертывающей скалярной функции $h$.

Удобное представление обратного Фурье преобразования произведения спектральных функций в круглых скобках последнего выражения в пространстве оригинала в виде свертки дает одно из представлений обратного преобразования Радона:

$$
\boldsymbol{f}\left(\boldsymbol{r}^{\prime}\right)=\int\left[\int h\left(\left(\boldsymbol{r}^{\prime} \cdot \boldsymbol{n}\right)-s\right) \boldsymbol{p}(s, \boldsymbol{n}) d s\right] \sin \theta d \boldsymbol{n}=\iint h(s) * \boldsymbol{p}(s, \theta, \alpha) \sin \theta d \theta d \alpha,
$$

где символ «*» - знак операции свертки; при этом

$$
h(s)=\int \frac{\omega^{2}}{(2 \pi)^{3}} e^{i \omega s} d \omega
$$

- свертывающая функция.

4. Реализация математического аппарата векторного преобразования Радона в магнитном неразрушающем контроле. На практике для описания физической величины, доступной косвенному измерению, используется определение магнитного потока индукции $\phi$ как совокупности (количества) силовых линий, проходящих сквозь рассматриваемую поверхность. Магнитный поток $\phi$ - величина скалярная и является интегралом вектора магнитной индукции $\boldsymbol{B}$ через конечную плоскость $\Omega$ первичного преобразователя:

$$
\phi=\int_{\Omega} \boldsymbol{B}(\boldsymbol{r}) d \boldsymbol{\sigma},
$$

где $d \boldsymbol{\sigma}=\boldsymbol{n} d \sigma$ - векторный элемент площади плоскости $\Omega$ с вектором нормали $\boldsymbol{n}$ :

$$
\phi(s, \boldsymbol{n})=\int_{\Omega, s}(\boldsymbol{B} \cdot \boldsymbol{n}) d \sigma ;
$$

скалярное произведение векторов $(\boldsymbol{B} \cdot \boldsymbol{n})$ равно потоку через элемент плоскости $\Omega$, которая удалена от центра координат на расстояние $s$. Скалярное произведение векторной функции индукции с единичным вектором тождественно равно проекции пр $\boldsymbol{n} B=(\boldsymbol{B} \cdot \boldsymbol{n}) /|\boldsymbol{n}|=(\boldsymbol{B} \cdot \boldsymbol{n})$.

Введем векторную функцию, ориентировав скаляр магнитного потока в направление вектора нормали:

$$
\boldsymbol{\phi}(s, \boldsymbol{n})=\phi(s, \boldsymbol{n}) \cdot \boldsymbol{n}
$$

тогда обратное векторное преобразование Радона для функции магнитной индукции:

$$
\boldsymbol{B}\left(\boldsymbol{r}^{\prime}\right)=\int_{0}^{\pi} \int_{0}^{\pi} h(s) * \phi(s, \theta, \alpha) \sin \theta d \theta d \alpha .
$$

\section{СПИСОК ЛИТЕРАТУРЫ}

1. Жильников A. А. Способ и алгоритмы магнитоиндукционного исследования ферромагнитных тел внутри объектов/ Дисс. на соиск. уч. степ. канд. техн. наук - Рязань, 2018.

2. Жильников А. А., Жильников Т. А., Жулев В. И. Концептуальная модель способа неразрушающего измерения магнитных полей внутри биологических объектов// Биомед. технол. радиоэлектроника. 2012. - № 7. - C. 37--43. 
3. Жильников А. А., Жильников Т. А., Жулев В. И. Квазистационарная модель описания магнитного поля при реализации способа магнитоиндукционного исследования ферромагнитных тел внутри объектов// Инж. физ. - 2017. - № 9. - С. 33--39.

4. Клюев В. В., Соснин Ф. Р., Ковалев А. В. Неразрушающий контроль и диагностика. - М.: Машиностроение, 2003.

5. Ландау Л. Д., Лифиии, Е. М. Теория поля. - М.: Наука, 1988.

6. Deans S. R. The Radon Transform and Some of Its Applications. - New York: Wiley, 1983.

Жильников Тимур Александрович

Академия права и управления ФСИН России, Рязань

E-mail: quadrus02@mail.ru

Маскина Мария Сергеевна

Академия права и управления ФСИН России, Рязань

E-mail: mariaya_maskina@mail.ru 